\title{
Prevalence of Anti-Phospholipid Autoantibodies and Their Association with Respiratory SOFA Component in Patients with COVID-19 Pneumonia: A Prospective Cohort Analysis in Tunisia
}

Amal ABOUDA ( $\square$ amal.abouda@fst.utm.tn )

Military Hospital of Tunis https://orcid.org/0000-0001-6336-4774

Yasmine BOUKHALFA

Military Hospital of Tunis

Wafa ANENE

Universite de Tunis El Manar Faculte de Medecine de Tunis

Zied HAJJEJ

Military Hospital of Tunis

Ezzeddine GHAZOUANI

Military Hospital of Tunis

Mustapha FERJANI

Military Hospital of Tunis

Iheb LABBENE

Military Hospital of Tunis

\section{Research}

Keywords: COVID-19, anti-cardiolipin, anti-phosphatidylserine, Sepsis-related Organ Failure Assessment, Respiratory Distress

Posted Date: July 12th, 2021

DOI: https://doi.org/10.21203/rs.3.rs-661812/v1

License: (c) (i) This work is licensed under a Creative Commons Attribution 4.0 International License. Read Full License 


\section{Abstract}

Purpose: The aim of our study was to evaluate the prevalence of aPLAs among Tunisian critically-ill covid19 and non-covid19 patients and to investigate the clinical significance of aPLAs by determining the SOFA score and their respiratory failure during their ICU stay.

Methods: We conducted a prospective observational cohort study including critically ill COVID-19 patients and non-COVID-19 patients with pulmonary origin sepsis, admitted to the intensive care unit. Blood samples were collected on days 1, 3, 5, 8 and 10 of hospitalization in order to measure titers of anticardiolipin (aCL), anti-phosphatidylserine (aPS) by chemiluminescence immunoassay.

Results: We enrolled 43 COVID-19 patients and 31 non COVID-19 with pulmonary origin sepsis. In-hospital mortality rate was significantly higher $(\mathrm{p}=0.026)$ in COVID-19 patients $(79 \%) .58 .8 \%$ of COVID-19 patients were aPLA positive; however, only $22.5 \%$ of the non-COVID-19 were positive for aPLA $(p=0.002)$.

A significant positive correlation existed between respiratory SOFA component at days $3,5,8$ and 10 and anti-phospholipid antibodies concentrations.

Conclusion: Based on our results, for the first time, anti-phospholipid antibodies may be used as an independent indicator of respiratory organ failure in critically ill patients, to stratify and assess the prognosis of pulmonary origin sepsis and COVID-19.

\section{Introduction}

The coronavirus disease 2019 is the largest public health emergency in recent times. New studies have shown that severe Corona-Virus Disease 2019 may be complicated by coagulopathy and disseminated intravascular coagulation [1]. On April 2021, Zhang et al. described three severely ill COVID-19 patients with circulating anti-phospholipid antibodies, suggesting for the first time that coagulopathy associated with COVID-19 could be an acquired thrombophilia close to the spectrum of anti-phospholipid syndrome (APS) [2]. Anti-phospholipid antibodies are auto-antibodies developed against phospholipid-binding proteins, such as Cardiolipin and Phosphatidylserine, that are expressed on pulmonary surfactant [3]. Pulmonary surfactant is produced by type-II pneumocytes, which express high levels of ACE2-receptors, first target of SARS-CoV2 [4]. During COVID19, pneumocytes necrosis leads to surfactant leakage exposing phospholipid proteins to the immune system [5]. Kanduc et al. found that the immune-reactive epitopes on the Spike glycoprotein of SARS-CoV2 share pentapeptides on human surfactant-related proteins, leading to auto-immunity via molecular mimicry [6].

Until this day, about 20 studies have reported a positive aPLA within COVID-19 patients [7]. A total cases of 1233 was analyzed and $54 \%$ were positive for at least one aPLA ( $a C L, \beta_{2} \mathrm{GPI}$, and/or LA) [8]. Both the high frequency and the diversity of aPLAs strongly suggest that these antibodies are actively produced during acute SARS-CoV2 infection. 
To our knowledge, the prevalence of aPLAs in severe cases of COVID-19 has never been undertaken in Tunisia. The aim of our study was to evaluate the prevalence of aPLAs among Tunisian critically-ill covid19 and non-covid19 patients and to investigate the clinical significance of aPLAs among the two groups of patients by determining the SOFA score and their respiratory failure during their ICU stay.

\section{METHODS}

\section{Study group}

In a prospective observational cohort study, we enrolled 74 critically ill patients with pneumonia referred to The Intensive Care Unit of the Military Hospital of Tunis. Two series of 43 COVID-19 pneumonia patients and 31 non-COVID-19 pulmonary origin septic patients were consecutively enrolled from October 2020 to May 2021. All COVID-19 patients had a confirmed COVID-19 diagnosis based on SARS-CoV2 detection by real-time RT-PCR RNA testing and by low dose computed tomography (CT) categorized according to expert consensus [9]. Non-COVID-19 patients with pulmonary origin sepsis were diagnosed according to sepsis-3 criteria [10]. All patients were without a clinical history of anti-phospholipid syndrome or autoimmune diseases. In case of pregnancy, age below 18 years old or high-dose immunosuppressive treatment, patients were excluded.

\section{Ethics statements}

This study complied with all relevant ethical regulations and was approved by the Local Personal Protection Ethics Committee of the Military Hospital of Tunis (date: 27-10-2020 number:

78bis/2020/CLPP/Hôpital Militaire de Tunis), which waived the requirement for informed consent given the discarded nature of the samples.

\section{Sampling}

Whole blood samples were collected in dry tubes on day 1, 3, 5, 8 and 10 of hospitalization by a trained hospital phlebotomist. Blood samples had been centrifuged at $2000 \mathrm{rpm}$ for 5 minutes. Serum was collected and divided into small aliquots tubes and then stored at $-40^{\circ} \mathrm{C}$ for subsequent assays.

\section{4- Clinical and biological data collection}

Clinical and biological data were obtained from the medical records of patients, including PaO2, FiO2, Platelets, creatinine, bilirubin, Glasgow Coma Scale, mean arterial pressure and noradrenalin dose.

\section{Measurement of aCL and aPS}

The enzyme immunoassay indirect-type ELISA was applied for the aCL and aPS highlighting to the three types of immunoglobulins IgM, IgG and IgA by commercial ELISA kits (EUROIMMUN®, Germany). The normal value is $\leq 12 \mathrm{RU} / \mathrm{mL}$ for all isotypes. Accordingly, patients having a concentration of aCL and/or aPS $>12 \mathrm{RU} / \mathrm{mL}$ at any time of the study were considered as aPLA positive patients, otherwise they were negative. 


\section{Severity assessment}

Severity of organ dysfunction was measured using the Sepsis-related Organ Failure Assessment (SOFA) score, which included respiration, coagulation, liver, cardiovascular and renal parameters. The cumulative SOFA score was calculated by the adding up of each item (range 0-24), and the higher the score was, the more extreme malfunction of the organ was [11].

Then, we calculated the respiratory-SOFA component according to the $\mathrm{PaO} 2 / \mathrm{FiO} 2$ ratio [11]. This component ranges from 0 to 4 and was recorded, as well as the cumulative SOFA-score, over the period of the study.

\section{Statistics}

Data were presented as number (percentages) for categorical variables and as means \pm SD if they were normally distributed, or as median values (IQR) if not, for continuous variables, to describe clinical and demographic characteristics.

Variables were tested for normality distribution by the Shapiro-Wilk test at a significance level of $5 \%$.

Clinical data were compared between the two groups ICU COVID-19 patients and ICU non-COVID-19 patients using the Khi2 test or the Fisher test for small numbers. Then, they were analyzed using either the Student $t$ test or the Mann-Whitney $U$ test, according to normality.

The paired t-student test was calculated to evaluate the evolution in timeline of the SOFA score, as well-as the respiratory SOFA component.

Linear correlation analysis between SOFA score and anti-phospholipid antibodies was gained by Pearson rank for normally distributed data, else Spearman rank. Differences were considered to be significant at a $p$ value $<0.05$. These analyses were performed using the IBM SPSS Statistics, version 22 (Armonk, USA). Graphics were sketched using Prism9.1.1 (GraphPad software, USA).

\section{Results}

\section{1-Patients Selection \& clinical characteristics}

From October 2020 to May 2021, we identified 205 patients with confirmed COVID-19 infection and 230 non-COVID-19 upon ICU admission.

There were 43 COVID-19 patients, eligible for study enrolment, aged $61.02 \pm 10.94$, of which 31 (72\%) were male and in $41.8 \%$ had diabetes mellitus. Of the 53 non-COVID-19 patients eligible, 31 were included; patients, which developed a pulmonary origin sepsis. They were aged $48.5 \pm 20.92,23(74.19 \%)$ were male and had diabetes mellitus in $51.61 \%$.

Demographic and clinical characteristics of the two groups are detailed in Table 1. 
Table 1

Demographic and clinical data between COVID and non-COVID groups

\begin{tabular}{|llll|}
\hline & ICU COVID-19 patients $(\mathbf{n}=$ & $\begin{array}{l}\text { ICU Non-COVID-19 patients }(\mathbf{n}= \\
\mathbf{3 1})\end{array}$ & $\mathbf{p}$-value \\
\hline Age, years, mean \pm SD & $61.02 \pm 10.94$ & $48.5 \pm 20.92$ & 0.275 \\
\hline Male, no (\%) & $31(72 \%)$ & $23(74.19 \%)$ & 0.841 \\
\hline Comorbidities & & $16(51.61 \%)$ & 0.406 \\
\hline Diabetes mellitus & $18(41.86 \%)$ & $3(9.67 \%)$ & 0.395 \\
\hline Dyslipidemia & $2(4.65 \%)$ & $15(48.38 \%)$ & 0.501 \\
\hline Hypertension & $17(39.5 \%)$ & $9(29.03 \%)$ & 0.111 \\
\hline $\begin{array}{l}\text { Chronic pulmonary } \\
\text { disease }\end{array}$ & $6(13.95 \%)$ & $3(9.67 \%)$ & 0.794 \\
\hline Chronic renal failure & $3(6.97 \%)$ & $20(64.51 \%)$ & 0.262 \\
\hline intubation & $24(55.81 \%)$ & $9(29.03 \%)$ & 0.601 \\
\hline Thromboembolic event & $17(39.53 \%)$ & $8.6 \pm 6.97$ & 0.364 \\
\hline ICU days, mean \pm SD & $19.86 \pm 9.78$ & $8.10 \pm 2.84$ & $0.005^{*}$ \\
\hline SOFA score admission & $8.44 \pm 2.87$ & $17(54.83 \%)$ & $0.026 *$ \\
\hline mortality & $34(79 \%)$ & & \\
\hline Data presented as n (\%), mean \pm SD. & & \\
\hline $\begin{array}{l}\text { COVID: corona virus disease ICU: intensive care unit, SOFA score: Sequential Organ Failure } \\
\text { Assessment score. }\end{array}$ & & \\
\hline
\end{tabular}

\section{High SOFA score in COVID-19 patients}

Significant difference was observed in mortality rate between COVID-19 patients (79\%) and non-COVID-19 patients $(54.83 \%)(p=0.026)$. Moreover, the SOFA score evolution was different between the two groups as shown in Fig. 1A. It was significantly higher in the COVID-19 patients group ( $8.44 \pm 2.87)$ than in nonCOVID-19 patients group $(7.47 \pm 2.61)$ on day $1(p=0.005)$, day3 $(9.56 \pm 3.63$ vs $6 \pm 3.76)$, day $5(12.44 \pm$ 4.64 vs $5.80 \pm 3.23)$, day8 $(12 \pm 4.61$ vs $4.53 \pm 3.50)$ and day $10(11.63 \pm 3.48$ vs $5.53 \pm 3.64)(p<0.0001$, respectively). The SOFA score increased from day 1 to day 10 in COVID-19 patients $(p=0.038)$. However, it decreased in non-COVID-19 patients (Fig. 1B), without reaching significance $(p=0.441)$.

\section{High Respiratory SOFA component in COVID-19 patients}

The second outcome is the observed severe respiratory dysfunction measured using the SOFA-respiratory component for the two groups. A severe respiratory distress was observed in the COVID-19 group 
illustrated in Fig. 2A.

On admission day, we registered a higher Respiratory component for the COVID-19 patients $(p=0.001)$, as well as day 3 , day 5 day 8 and day 10 ( $p<0.0001$, respectively).

The respiratory-SOFA component decreased from day 1 to day 10 in non-COVID-19 patients $(p<0.0001)$, but not in COVID-19 patients ( $p=0.267$ ) (Fig. 2B).

\section{2-Prevalence of aPLA in ICU critically ill patients}

Table 2 shows that the prevalence of aPLA antibodies was significantly higher in the COVID-19 group $(58.1 \%)$ than the non-COVID-19 pulmonary-origin sepsis group $(22.5 \%)(p=0.002)$.

Table 2

aPLAs in the COVID and non-COVID groups

\begin{tabular}{|c|c|c|c|}
\hline & $\begin{array}{l}\text { ICU COVID-19 patients }(n= \\
\text { 43) }\end{array}$ & $\begin{array}{l}\text { ICU Non-COVID-19 patients }(n= \\
\text { 31) }\end{array}$ & $p$-value \\
\hline $\begin{array}{l}\mathrm{aCL} \lg M G A, \text { no, } \\
(\%)\end{array}$ & $25(58.13 \%)$ & $6(19.35 \%)$ & $0.001 * \star$ \\
\hline $\begin{array}{l}\text { aPS IgMGA, no, } \\
(\%)\end{array}$ & $10(23.25 \%)$ & $3(9.67 \%)$ & 0.130 \\
\hline All aPLA, no, (\%) & $25(58.1 \%)$ & $7(22.5 \%)$ & $0.002 * *$ \\
\hline \multicolumn{4}{|c|}{ Data presented as $\mathrm{n}(\%)$, mean \pm SD. } \\
\hline \multicolumn{4}{|c|}{$\begin{array}{l}\text { aCL: anti-Cardiolipin antibodies, aPS : anti-Phosphatidylserine antibodies, aPLA : antiphospholipids } \\
\text { IgMGA : combined isotypes }\end{array}$} \\
\hline
\end{tabular}

Indeed, in the COVID-19 group, there were 25 (58.1\%) tested positive for the aCL IgMGA. While, in the nonCOVID-19 group, only 6 (19.35\%) patients were positive for aCL IgMGA. Figure 3A illustrated this significant difference at all time-points on day 1 , day3, day 5 , day8 and day 10 ( $p<0.0001$, respectively).

We compared also the aPS production between the two groups. Ten COVID-19 patients (23.2\%) vs three non COVID-19 pulmonary-origin sepsis patients (9.67\%) were tested positive for the aPS IgMGA. We observed a significantly higher prevalence of aPS production in COVID-19 group compared to non-COVID19 group at all time-points ( $p<0.0001$, respectively) (Fig. 3B).

\section{3- Association of aPLA with respiratory organ failure}

As well as in non-COVID-19 pulmonary origin sepsis group, the level of aPS at day 3 was correlated with the respiratory-SOFA component of the same day of measurement in COVID-19 group $(r=0.385, p=$ 0.032 , for each group). For both groups, the aCL and aPS titers at day 5 were correlated with the respiratory-SOFA component at day $5(r=0.490, p=0.005 ; r=0.399, p=0.026$, respectively). 
In the COVID-19 group, the correlation was slightly stronger, observed between the aCL and aPS at day 8 with the respiratory-SOFA component of the same day $(r=0.634, p<0.0001 ; r=0.633, p<0.0001)$, than do the correlation between these parameters in non-COVID-19 group $(r=0.508, p=0.004 ; r=0.634, p<$ 0.0001).

Besides, at day 10, the correlation between the two types of anti-phospholipid (aCL and aPS autoantibodies) and the respiratory SOFA component was maintained for both groups $(r=0.633, p<$ 0.0001).

In timeline, the respiratory SOFA component at day 5 predict the elevation in titers of aCL and aPS at the eighth day in COVID-19 patients $(r=0.634, p<0.0001 ; r=0.633, p<0.0001)$ and in non-COVID-19 patients $(r=0.508, p=0.004 ; r=0.634, p<0.0001)$. It was also correlated with $a C L$ and aPS production at day 10 $(r=0.633, p<0.0001 ; r=0.622, p<0.0001$ in non-COVID-19 group vs $r=0.465, p=0.008 ; r=0.421, p=$ 0.018 in COVID-19 group).

Moreover, the correlation in different timelines continued to describe the prediction of the aCL and aPS production at day 10 by the respiratory SOFA component measurement at day $8(r=0.633, p<0.0001 ; r=$ $0.622, p<0.0001$, respectively for each group).

\section{Discussion}

COVID-19-induced-APS-likesyndrome is a new term [12], as many studies reported a priori autoantibody production in severe-COVID-19 patients $(13), 15]$. Their prevalence is balanced among studies. A prospective observational study performed at a third level hospital in Madrid, revealed that only $8.3 \%$ of the patients were positive for anticardiolipin IgM and anti-ß2-glycoprotein-I IgM [16]. On the contrary, Zuo et al. found that $52 \%$ of their patients tested positive for aPLA [17].

Until now, the incrimination of aPLA in the coagulopathy linked to COVID-19 still controversial. These autoantibodies may occur in critically ill patients following different infections, such as sepsis $[2,18]$.

In our study, we enrolled 43 critically ill COVID-19 patients and 31 critically ill non-covid19 patients with pulmonary origin sepsis, admitted in the intensive care unit in the military hospital of Tunis between October 2020 and May 2021. All patients were matched by age, sex, and comorbidities $(p>0.05)$.

We found a slightly significant higher rate of mortality in COVID-19 patients $(79 \%)$ compared to septic non-COVID-19 patients $(54.83 \%)(p=0.026)$.

The SOFA-score was calculated among the groups to evaluate quantitatively and objectively the degree of organ failure through several parameters including the oxygenation efficiency, mean arterial pressure, creatinine or urine volume, bilirubin, platelets and Glasgow Coma Scale (GCS) for the respiratory, circulatory, renal, hepatogenic, coagulation and neurologic systems, respectively [11]. Prospective evaluations have validated the SOFA score as a usefull tool for the assessment of morbidity in critical illness [19]. 
The current study showed that the SOFA-score was dramatically higher in the COVID-19 patients group $(8.44 \pm 2.87)$ than in non-COVID-19 patients group on day $1(7.47 \pm 2.61)(p=0.005)$, day 3 , day 5 , day 8 and day 10 ( $p<0.0001)$, inferring aggravating conditions in the COVID-19 patients. Indeed, Sijia Liu et al. reported that a SOFA-score $\geq 3$ is associated with mortality in critically ill COVID19 patients. This score is a highly sensitive marker of in-hospital mortality [20].

In respiratory infections, hypoxic conditions are typical due to decreased oxygen inhalation [21]. Indeed, we observed severe respiratory dysfunction measured using the SOFA-respiratory component between the two groups on the admission day, day 3 , day 5 , day 8 and day 10 . Assessment of the respiratory SOFAcomponent relies on invasive arterial monitoring to measure arterial partial pressure of oxygen followed by calculation of the $\mathrm{PaO} 2 / \mathrm{FiO} 2$ ratio which refers to the oxygenation index and leads to SOFA score variation [22]. The specific SOFA score ranges from 0 to 4 . This score was significantly higher in COVID$19(\geq 2)$ on day $1(p=0.001)$ to day $10(p<0.0001)$, than in non COVID-19 patients $(<2)$.

Furthermore, its evolution among time was significantly decreased in non-COVID-19 pulmonary origin sepsis patients $(p<0.0001)$ between day $1(1.29 \pm 1.16)$ and day $10(0.41 \pm 0.71)$. Conversely, the evolution of respiratory SOFA-component in COVID-19 kept high levels between day $1(2.6 \pm 1.66)$ and day $10(2.93 \pm 1.38)$, and no decrease has been registered $(p=0.304)$.

This result indicates an exaggerated respiratory distress and lung injury in SARS-CoV2-viral pneumonia patients compared to bacterial pneumonia in non-COVID-19 septic patients.

Different studies have shown that SARS-CoV-2 infection induces down-regulation of surfactant proteins and an increase in cell death [23]. SARS-CoV-2 infects alveolar epithelial cells by recognizing the ACE2 receptor [24]. Following the virus internalization, the exfoliation of ACE2 reduces the expression of ACE2 on the cell membrane which weakens its lung-protective effects $[25,26]$. This may lead to alveolar epithelial cell necrosis and aggravation of lung inflammation [26].

In our study, we measured aCLIgMGA and aPSIgMGA autoantibodies in critically ill COVID-19 and nonCOVID-19 patients, at different time points during their hospitalization in ICU.

We found $58.1 \%$ of COVID-19 patients were positive for at least one antibody based on the manufacturer's cutoff. A striking difference was observed between the autoantibody profiles in COVID-19 patients as compared to the one in non-COVID-19 concerning the titers of aPLA. The vast majority of positive results were aCL IgMGA (58.13\% COVID-19 vs $16.98 \%$ non COVID-19), rather than aPS IgMGA ( $23.25 \%$ covid-19 vs $7.5 \%$ non-COVID-19).

This high occurrence of aPLA autoantibodies in patients with COVID-19 could be explained by the high risk for thromboembolic events. Indeed, Hui Shi et al. described the endothelial cell activation as a part of the COVID-19 thrombo-inflammatory storm. They reported that circulating aPLA may be predictors of endothelial cell activation in COVID-19 and their depletion restrained the upregulation of E-Selectin, VCAM-1 and ICAM-1, the three markers of endothelial cell activation [28]. 
Interestingly, we detected modest correlations between anticardiolipin, anti-PS autoantibodies and the respiratory SOFA component of the same day of measurement for both groups. We noted that the correlation was slightly stronger, at day $8(r=0.634, p<0.0001 ; r=0.633, p<0.0001)$, in the COVID-19 group. This may suggest a local production of aPLA in lungs.

Moreover, the respiratory-SOFA component on day 5 was strongly and significantly correlated to aPLA titers on day 8 and 10 for both groups. This led us to think that the respiratory SOFA-component can predict the increase in the anticardiolipin and antiphosphatidylserine antibodies.

In the context of major cellular distress and tissue injury, the system may be overwhelmed and mitochondrial Damage-Associated Molecular Pattern molecules (DAMPs) are released into the cytoplasm [28], triggering a pro-inflammatory response. This led us to think about a production of anti-phospholipid antibodies against cardiolipin located in the internal membrane of mitochondria [29], which escaped the autophagy due to a dysregulated immune response [30].

To summarize, our study clarifies, at least in part, the kinetics of aPLA synthesis in COVID-19 and in pulmonary sepsis and supports the use of aPLA autoantibodies as predictors of a potential harmful increase in the respiratory SOFA component. The aPLA assays had a good prognostic efficiency, suggesting that measuring aCL and aPS might be used as a secondary criterion for determining severity.

\section{Conclusion}

The overwhelming power of this extremely feasible assay lies in its inclusion of the effect of aPLA production on the respiratory organ failure, as well as in the determination of the time of its production. As it may be a relevant biomarker, the development of aPLA in response to therapy is of clinical significance.

\section{Abbreviations}

aPLA

anti-phospholipid

$\mathrm{aCL}$

anti-cardiolipin

aPS

anti-phosphatidylserine

SOFA

Sepsis-related Organ Failure Assessment

ICU

Intensive Care Unit

COVID-19

Coronavirus Disease of 2019 


\section{Declarations}

\section{Ethics approval and consent to participate}

This study complied with all relevant ethical regulations and was approved by the Local Personal Protection Ethics Committee of the Military Hospital of Tunis (date: 27-10-2020 number: 78bis/2020/CLPP/Hôpital Militaire de Tunis).

\section{Consent for publication}

Written informed consent was obtained from all patients or next of kin before inclusion.

\section{Availability of supporting data}

The datasets used and/or analysed during the current study are available from the corresponding author on reasonable request.

\section{Competing interests}

The authors declare that they have no competing interests.

\section{Funding}

The Tunisian Ministry of Higher Education, Research, and Technology, Laboratory of Hemodynamic Reanimation and Dialysis Technics (LR12DN01) and the Tunisian Ministry of Defense supported this work.

\section{Authors' contributions}

AA designed the study, performed the anti-phospholipid measurement and statistical analysis, and was a major contributor in writing the manuscript. YB provided substantial contributions in data curation and writing the original draft. All authors contributed to drafting of the work, revising it critically for important intellectual content.

\section{Acknowledgements}

We would like to thank all of the staff at the medical intensive care unit and the Laboratory of Immunology at the Military Hospital of Tunis, Tunisia.

\section{References}

1. Yin S, Huang M, Li D, Tang N. Difference of coagulation features between severe pneumonia induced by SARS-CoV2 and non-SARS-CoV2. J Thromb Thrombolysis mai. 2021;51(4):1107-10.

2. Zhang Y, Xiao M, Zhang S, Xia P, Cao W, Jiang W, et al. Coagulopathy and Antiphospholipid Antibodies in Patients with Covid-19. N Engl J Med 23 avr. 2020;382(17):e38. 
3. Giannakopoulos B, Passam F, Rahgozar S, Krilis SA. Current concepts on the pathogenesis of the antiphospholipid syndrome. Blood 15 janv. 2007;109(2):422-30.

4. Carcaterra M, Caruso C. Alveolar epithelial cell type II as main target of SARS-CoV-2 virus and COVID19 development via NF-Kb pathway deregulation: A physio-pathological theory. Med Hypotheses janv. 2021;146:110412.

5. Mendoza-Pinto C, García-Carrasco M, Cervera R. Role of Infectious Diseases in the Antiphospholipid Syndrome (Including Its Catastrophic Variant). Curr Rheumatol Rep oct. 2018;20(10):62.

6. Kanduc D, Shoenfeld Y. On the molecular determinants of the SARS-CoV-2 attack. Clin Immunol Orlando Fla juin. 2020;215:108426.

7. Taha M, Samavati L. Antiphospholipid antibodies in COVID-19: a meta-analysis and systematic review. RMD Open mai. 2021;7(2):e001580.

8. Siguret V, Voicu S, Neuwirth M, Delrue M, Gayat E, Stépanian A, et al. Are antiphospholipid antibodies associated with thrombotic complications in critically ill COVID-19 patients? Thromb Res nov. 2020;195:74-6.

9. Simpson S, Kay FU, Abbara S, Bhalla S, Chung JH, Chung M, et al. Radiological Society of North America Expert Consensus Document on Reporting Chest CT Findings Related to COVID-19: Endorsed by the Society of Thoracic Radiology, the American College of Radiology, and RSNA. Radiol Cardiothorac Imaging. 1 avr 2020;2(2):e200152.

10. Singer M, Deutschman CS, Seymour CW, Shankar-Hari M, Annane D, Bauer M, et al. The Third International Consensus Definitions for Sepsis and Septic Shock (Sepsis-3). JAMA 23 févr. 2016;315(8):801.

11. Vincent J-L, Moreno R, Takala J, Willatts S, De Mendonça A, Bruining H, et al. The SOFA (Sepsisrelated Organ Failure Assessment) score to describe organ dysfunction/failure. Intensive Care Med 1 juill. 1996;22(7):707-10.

12. Karahan S, Erol K, Yuksel RC, Artan C, Celik I. Antiphospholipid antibodies in COVID-19-associated pneumonia patients in intensive care unit. Mod Rheumatol. 1 mars 2021;1-10.

13. Pavoni V, Gianesello L, Horton A. Antiphospholipid antibodies in critically ill COVID-19 patients with thromboembolism: cause of disease or epiphenomenon? J Thromb Thrombolysis.10 mai 2021; 111: 33973157.

14. Borghi MO, Beltagy A, Garrafa E, Curreli D, Cecchini G, Bodio C, et al. Anti-Phospholipid Antibodies in COVID-19 Are Different From Those Detectable in the Anti-Phospholipid Syndrome. Front Immunol 15 oct. 2020;11:584241.

15. Amezcua-Guerra LM, Rojas-Velasco G, Brianza-Padilla M, Vázquez-Rangel A, Márquez-Velasco R, Baranda-Tovar F, et al. Presence of antiphospholipid antibodies in COVID-19: a case series study. Ann Rheum Dis mai. 2021;80(5):e73-3.

16. Galeano-Valle F, Oblitas CM, Ferreiro-Mazón MM, Alonso-Muñoz J, del Toro-Cervera J, di Natale M, et al. Antiphospholipid antibodies are not elevated in patients with severe COVID-19 pneumonia and venous thromboembolism. Thromb Res août. 2020;192:113-5. 
17. Zuo Y, Estes SK, Gandhi AA, Yalavarthi S, Ali RA, Shi H, et al. Prothrombotic antiphospholipid antibodies in COVID-19. Preprint at https://www.medrxiv.org/content/10.1101/2020.06.15.20131607v2 (2020).

18. Vassalo J, Spector N, Meis E de, Soares M, Salluh JIF. Antiphospholipid antibodies in critically ill patients. Rev Bras Ter Intensiva juin. 2014;26(2):176-82.

19. Ferreira FL. Serial Evaluation of the SOFA Score to Predict Outcome in Critically III Patients. JAMA. oct 2001;10(14):1754. 286(.

20. Liu S, Yao N, Qiu Y, He C. Predictive performance of SOFA and qSOFA for in-hospital mortality in severe novel coronavirus disease. Am J Emerg Med oct. 2020;38(10):2074-80.

21. Schaible B, Schaffer K, Taylor CT. Hypoxia, innate immunity and infection in the lung. Respir Physiol Neurobiol déc. 2010;174(3):235-43.

22. Lambden S, Laterre PF, Levy MM, Francois B. The SOFA score-development, utility and challenges of accurate assessment in clinical trials. Crit Care déc. 2019;23(1):374.

23. Islam ABMMdK, Khan MdA-A-K. Lung transcriptome of a COVID-19 patient and systems biology predictions suggest impaired surfactant production which may be druggable by surfactant therapy. Sci Rep déc. 2020;10(1):19395.

24. Zhang $\mathrm{H}$, Penninger JM, Li Y, Zhong N, Slutsky AS. Angiotensin-converting enzyme 2 (ACE2) as a SARS-CoV-2 receptor: molecular mechanisms and potential therapeutic target. Intensive Care Med avr. 2020;46(4):586-90.

25. Ziegler CGK, Allon SJ, Nyquist SK, Mbano IM, Miao VN, Tzouanas CN, et al. SARS-CoV-2 Receptor ACE2 Is an Interferon-Stimulated Gene in Human Airway Epithelial Cells and Is Detected in Specific Cell Subsets across Tissues. Cell mai. 2020;181(5):1016-35.e19.

26. Imai Y, Kuba K, Rao S, Huan Y, Guo F, Guan B, et al. Angiotensin-converting enzyme 2 protects from severe acute lung failure. Nature juill. 2005;436(7047):112-6.

27. Shi H, Zuo Y, Navaz S, Harbaugh A, Hoy C, Gandhi AA, et al. Endothelial cell-activating antibodies in COVID-19. Preprint at https://www.medrxiv.org/content/10.1101/2021.01.18.21250041v3 (2021).

28. Grazioli S, Pugin J. Mitochondrial Damage-Associated Molecular Patterns: From Inflammatory Signaling to Human Diseases. Front Immunol. 2018;9:832.

29. Pizzuto M, Pelegrin P. Cardiolipin in Immune Signaling and Cell Death. Trends Cell Biol nov. 2020;30(11):892-903.

30. Mehrzadi S, Karimi MY, Fatemi A, Reiter RJ, Hosseinzadeh A. SARS-CoV-2 and other coronaviruses negatively influence mitochondrial quality control: beneficial effects of melatonin. Pharmacol Ther août. 2021;224:107825.

\section{Figures}




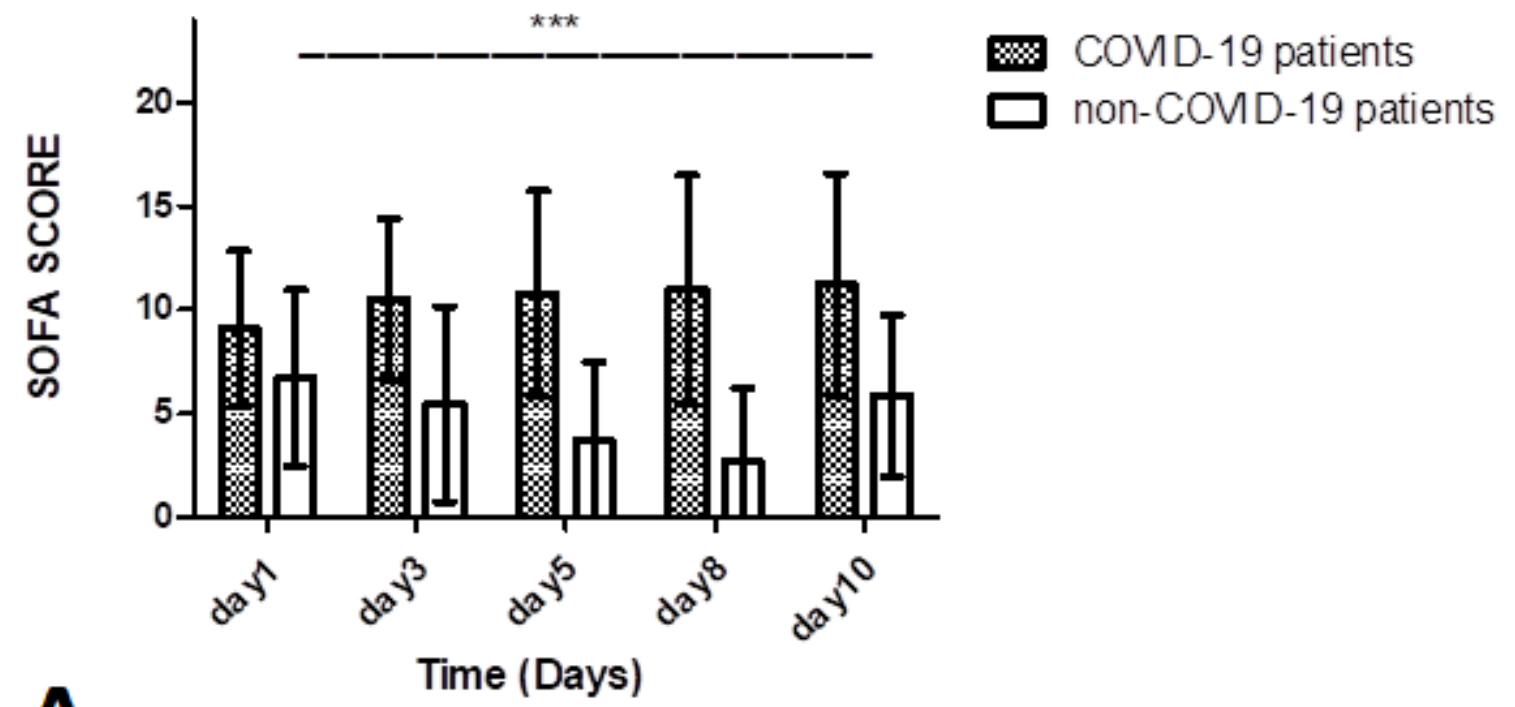

A

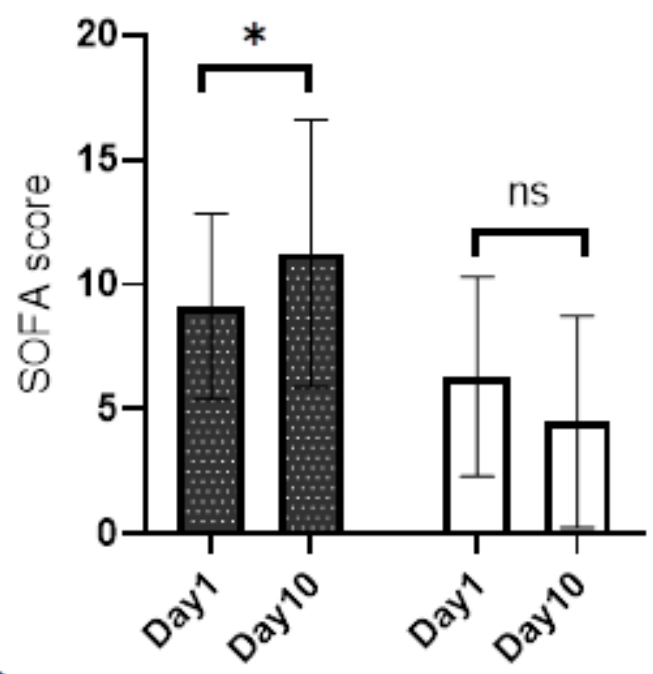

m COVID-19 patients

口 non-COVID-19 patients

B

Figure 1

A: SOFA score evolution among COVID and non-COVID groups hospitalized in ICU from day 1 to day 10 . SOFA score was calculated in means $\pm S D$. ${ }^{* *} p<0.05$ ICU COVID-19 patients versus ICU non-COVID-19 patients B: Increase of SOFA score in COVID-19 patients between day 1 and day 10. SOFA score was calculated in means $\pm S D$. ${ }^{\star \star} p<0.05$ day 1 versus day 10 


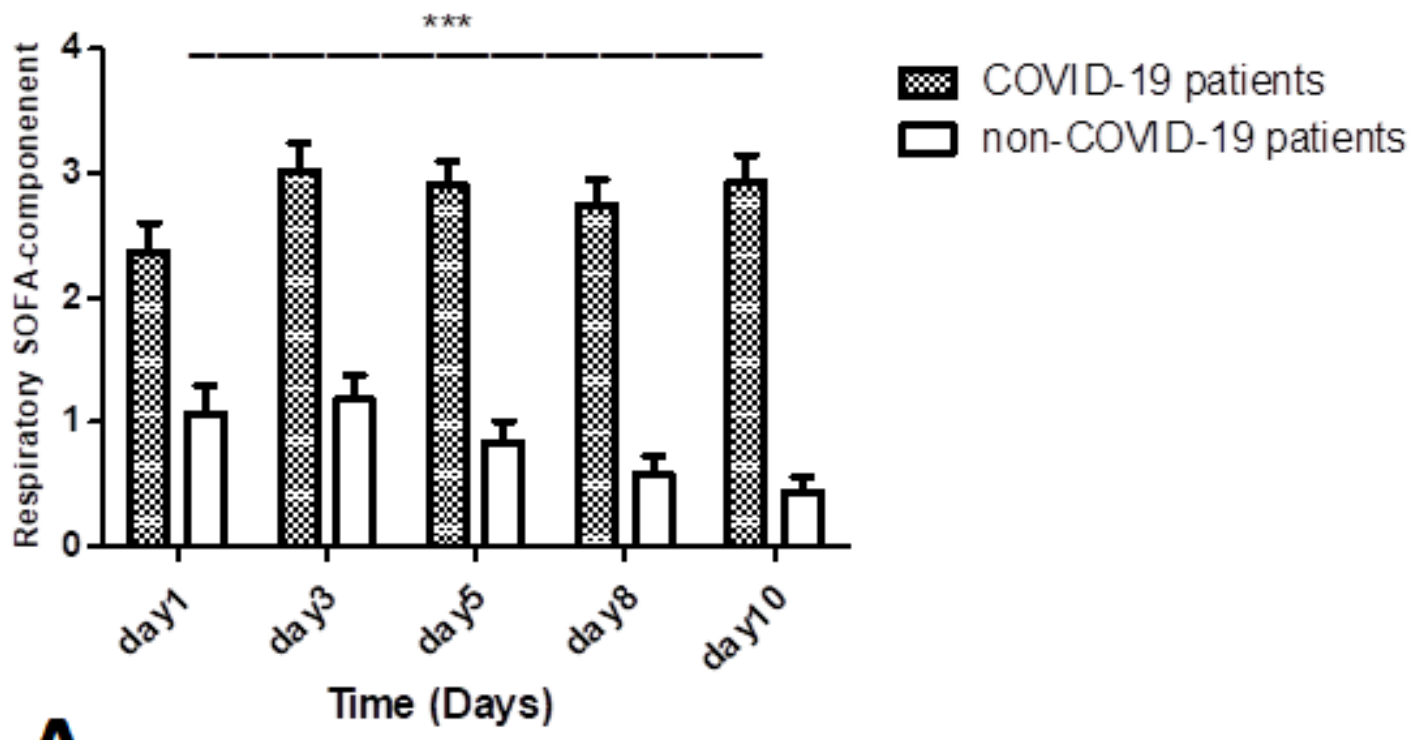

A

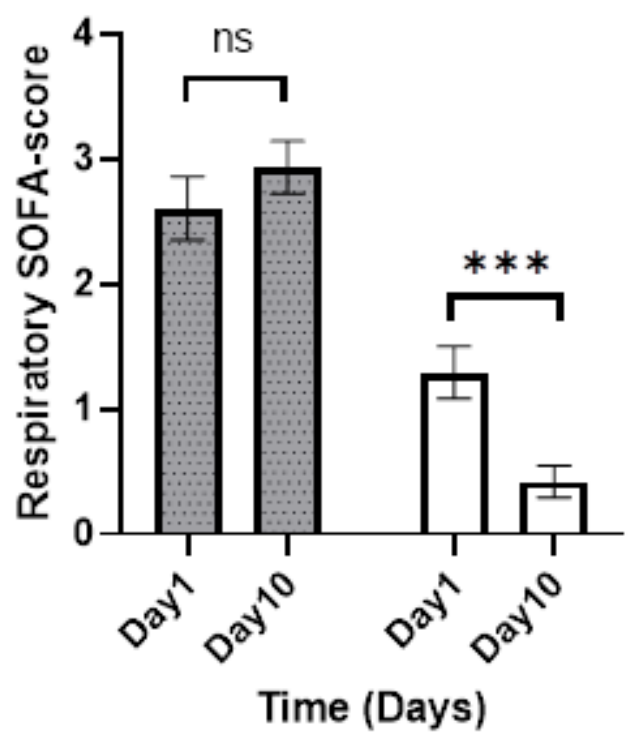

COVID-19 patients

$\square$ non-COVID patients

B

Figure 2

A: Respiratory SOFA component evolution among COVID and non-COVID groups hospitalized in ICU from day 1 to day 10. Respiratory SOFA score was calculated in means \pm SD. ${ }^{* \star} p<0.05$ ICU COVID-19 patients versus ICU non-COVID-19 patients B: Increase of SOFA score in COVID-19 patients between day 1 and day 10 . SOFA score was calculated in means \pm SD. ${ }^{\star \star} p<0.05$ day 1 versus day 10 


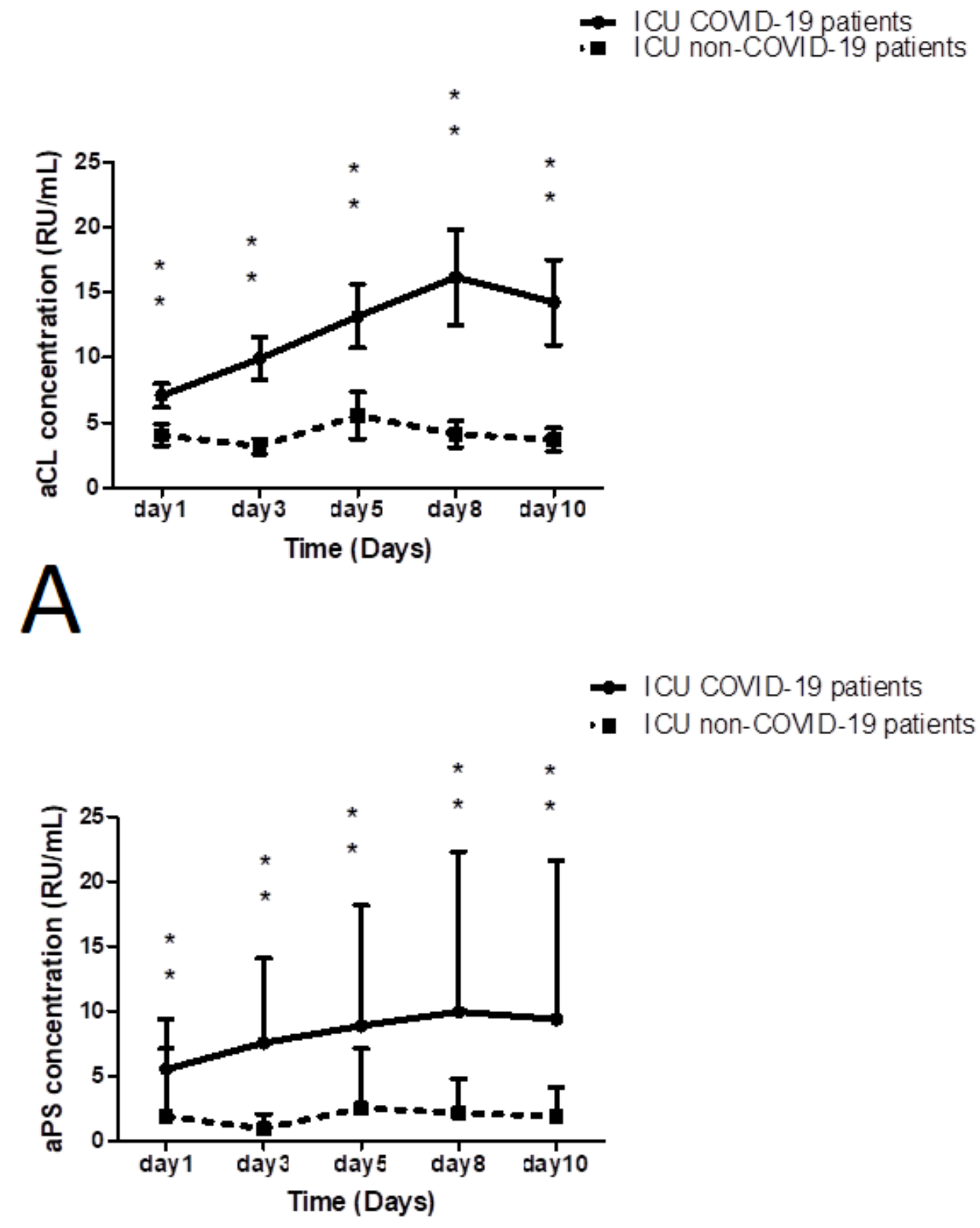

B

Figure 3

A: Comparison of the evolution of anti-cardiolipin antibodies between the COVID-19 and non-COVID-19 groups hospitalized in ICU, during the period study, from day 1 to day $10 .{ }^{* *} p<0.05$ ICU COVID-19 patients versus ICU non-COVID-19 patients B: Comparison of the evolution of anti-phosphatidylserine antibodies between the COVID-19 and non-COVID-19 groups hospitalized in ICU, during the period study, from day 1 to day 10. ${ }^{* *}$ p $<0.05$ ICU COVID-19 patients versus ICU non-COVID-19 patients 


\section{Supplementary Files}

This is a list of supplementary files associated with this preprint. Click to download.

- Authorstatement.docx

- Highlights.docx

- ScanaccordethiqueCOVIDPL.pdf 